Pacific Journal of Mathematics

QUELQUES CLASSES DE COBORDISME NON ORIENT Er

A. DIDIERJEAN 


\title{
QUELQUES CLASSES DE COBORDISME NON ORIENTE REFUSANT DE SE FIBRER SUR DES SPHERES
}

\section{A. DidierJeAN}

\begin{abstract}
Conner and Floyd introduced the problem of finding which cobordism classes can be represented by the total space of a bundle over a given sphere. We consider here the case of cobordism classes with dimension smaller than the double of the dimension of the given sphere.
\end{abstract}

1. Introduction. Dans l'étude du problème posé par Conner et Floyd ([2]), á savoir, quelles sont les classes de cobordisme non orienté qui se fibrent sur des sphères, apparait deux types de conditions nécéssaires.

En plus de conditions de relations sur les nombres caractéristiques ([1], [4]), intervient une condition naturelle d'existence d'applications dont la fibre théorique a une homologie finie ([3]).

Si les premières de ces conditions nécessaires permettent de donner des résultats, parfois complets, dans le cas des sphères $S^{k}, k \leq 8$ ([2], $[1],[5],[4])$, ce deuxième type de condition épuise le cas de toutes petites fibres sur des grandes sphères ([3]).

Dans ces deux cas les algèbres $A^{*}(\omega)$, introduites par R. E. Stong ([6]) et utilisées dans [3] et [4] s'avèrent un outil très utile. Ici, utilisant une démonstration de R. E. Stong ${ }^{1}$, on démontre que pour toute classe de cobordisme non nulle, pour toute variété $X$ de cette classe et toute application $f: X \rightarrow S^{k}$, la fibre théorique de cette application $f$ a une homologie non finie si $p<(k-2) / 2$.

Enfin, au moyen d'une algèbre $A^{*}$ reliant les classes de la fibre á celles de l'espace total du fibré considéré, des résultats partiels sont donnés pour $p<k$.

Dans ce qui suit, seul le cas "non orienté" étant considéré, $H^{*}($ ) désigne la cohomologie á coeffiscients dans le corps $\mathbf{Z} / 2 \mathbf{Z}$, la lettre $\omega$ désigne les classes de cobordisme non orienté, $N_{n}$ le groupe formé de ces classes.

Je tiens á remercier vivement le Cinvestav. de l'I.P.N. de Mexico pour son acceuil durant l'année scolaire 1986/87.

\footnotetext{
${ }^{1}$ Dans [3], la Proposition 2.4, enonce par erreur etait en fait non demontree. La demonstration de ce resultat, presente ici, m'a ete envoye par R. E. Stong.
} 
2. Quelques propriétés des algèbres de classes caractéristiques invariantes par cobordisme. Pour toute classe de cobordisme $\omega \in N_{n}$, $A^{*}(\omega)$ désigne son algèbre de classes caractéristiques "invariantes par cobordisme", construite comme suit ([6], [3]):

Pour toute variété $X$ de la classe $\omega$, on considère l'idéal des classes caractéristiques n'intervenant que dans des nombres caractéristiques nuls de la classe $\omega$ :

$$
I^{l}(\omega)=\left\{\alpha \in H^{l}\left(G_{n}\right) / \text { si } l \leq n, \forall \beta \in H^{n-l}\left(G_{n}\right), f^{n}(\alpha \beta)=0\right\}
$$

où $G_{n}$ désigne la grassmannienne des plans de dimension $n$ de $\mathbf{R}^{\infty}$ et $f: X \rightarrow G_{n}$ une application classifiante du fibré tangent de la variété $X$. Alors:

$$
A^{*}(\omega)=H^{*}\left(G_{n}\right) / I^{*}(\omega)
$$

L'algèbre $A^{*}(\omega)$ est une algèbre de Poincaré avec action de l'algèbre de Steenrod ([6]) et s'identifie pour toute variété de la classe $\omega$ à un quotient de la sous algèbre de ses classes de Stiefel Whitney. En particulier si pour un cran $k, A^{k}(\omega) \neq 0$, pour toute variété $X \in \omega$, on aura $H^{k}(X) \neq 0$.

Proposition (2.1). Soit $\omega \in N_{p+k}$ avec $p<(k-2) / 2$, ou $p=(k-2) / 2$ si $p$ est impair, si pour tout $j, p+1<j<k-1$, on $a, A^{j}(\omega)=0$, alors $\omega=0$.

Démonstration (R. E. Stong). Dans un premier temps on démontre que sous les hypothèses précédentes, l'algèbre $A^{*}(\omega)$ est engendré par les seuls éléments $w_{1}, \ldots, w_{p+1}$.

-Si $2 p+2<k$ soit $v$ la classe de $\mathrm{Wu}$ de $\omega$ dans $A^{*}(\omega)$. Comme $A^{*}(\omega)=0$ pour $p+1<j<k-1$, on a:

$$
v=1+\cdots+v_{[(k+p) / 2]}=1+\cdots+v_{p+1} \text { et } \quad w_{p+2}=\cdots=w_{k-2}=0
$$

Or $w_{q}=\sum_{i+j=q} \operatorname{Sq}^{i}\left(v_{j}\right), j \leq p+1$, les seuls éléments éventuellement non nuls sont donc obtenus pour $q \leq 2 j \leq 2 p+2$.

Comme de plus $k>2 p+2$, la seule classe $w_{q}$ qui peut être non nulle pour $q>p+1$ est $w_{k-1}$ pour $k-1=2 p+2$.

Alors $w_{k-1}=w_{2 p+2}=\mathrm{Sq}^{p+1}\left(v_{p+1}\right)=v_{p+1}^{2}$ est bien un élément de l'algèbre engendré par $w_{1} \ldots w_{p+1}$. 
-Si $2 p+2=k$ et $p$ est impair, les seules classes $w_{q}$ éventuellement non nulles pour $q>p+1$ sont $w_{k-1}$ et $w_{k}=w_{2 p+2}$, et l'on a:

$$
\begin{gathered}
w_{k-1}=\mathrm{Sq}^{p}\left(v_{p+1}\right)=\mathrm{Sq}^{1} \mathrm{Sq}^{p-1}\left(v_{p+1}\right), \quad \text { car } p \text { est impair } \\
\text { Or Sq} \\
w_{k}=\mathrm{Sq}^{p+1}\left(v_{p+1}\right) \in A^{2 p}=A^{k-2}=0 \text {, d'où } w_{k-1}=0 \\
\left.v_{p+1}\right)=v_{p+1}^{2} \text { est bien dans l'algèbre engendré }
\end{gathered}
$$

par les classes $w_{1} \ldots w_{p+1}$.

Pour terminer il faut démontrer que les conditions de nullité pour $A^{j}(\omega)$ et la petite valeur des degrés des générateurs $w_{q}$, implique la nullité de tous les nombres caractéristiques de la classe $\omega$.

-Si $2 p+2<k-1$ pour tout nombre caractéristique de $\omega$, $w_{1}^{i_{1}} \cdots w_{p+1}^{i_{p+1}}$, on considère $w_{1}^{S_{1}} \cdots w_{p+1}^{s_{p+1}}$ un diviseur de degré $\alpha=\sum j s_{j}$ le plus grand possible, avec $\alpha \leq p+1$.

Alors tout multiple de la forme $w_{1}^{s_{1}} \cdots w_{p+1}^{s_{p+1}} \cdot w_{i}$ est nul, car il appartient á $A^{\alpha+i}(\omega)=0$ pour $p+1<\alpha+i<2 p+2<k-1$.

-Si $2 p+2=k-1$ le seul nombre caractéristique n'ayant pas de diviseurs dans la tranche nulle pour $A^{j}(\omega)$ est $\left(w_{p+1}\right)^{3}$. Dans ce cas l'algèbre $A^{*}$ est engendrée par le seul élément $w_{p+1}$ et donc $v_{1}=v_{2}=$ $\cdots=v_{p}=0, v_{p+1}=w_{p+1}$.

Ainsi $\left(w_{p+1}\right)^{3}=\left(v_{p+1}\right)^{3}=\mathrm{Sq}^{p+1}\left(w_{p+1}^{2}\right)=\left[\mathrm{Sq}^{(p+1) / 2}\left(w_{p+1}\right)\right]^{2}$ si $p+1$ est pair et zéro autrement.

Or pour $p+1$ pair $A^{(3 p+3) / 2}(\omega)=A^{(k+p) / 2}(\omega)=0$. Tous les nombres caractéristiques de $\omega$ sont donc bien nuls.

-Si $2 p+2=k$, les seuls éléments de $A^{*}(\omega)$ qui peuvent être non nuls sont $w_{p}, w_{p+1}$ et $\left(w_{p+1}\right)^{2}$.

Le seul nombre éventuellement non nul de $\omega$ est donc $w_{p} \cdot\left(w_{p+1}\right)^{2}$. Ainsi dans $A^{*}(\omega)$ on a:

$$
w_{1}=w_{2}=\cdots=w_{p-1}=0, \quad v_{1}=v_{2}=\cdots=v_{p-1}=0, \quad \text { et } v_{p}=w_{p} \text {. }
$$

D'où $w_{p}\left(w_{p+1}\right)^{2}=\mathrm{Sq}^{p}\left((w)^{2}\right)=0$ par la formule de Cartan, l'entier $p$ étant impair.

Si á présent $f: X^{k+p} \rightarrow S^{k}$ est un fibré $C^{\infty}$ de fibre $F$, connexe, la classe de son espace total $X$ dans le groupe de cobordisme $N_{k+p}$ (resp. de sa fibre $F$ dans le groupe $N_{p}$ ) sera noté $[X]$ (resp., $[F]$ ).

Pour $p$ fixé, si $I^{n}$ désigne une partition de l'entier $n$, parmis des entiers inférieurs à $p, w^{I^{n}}$ le monome correspondant á cette partition, l'ensemble de ces monomes $\left\{w^{I^{n}}\right\}$ constitue une base du module $H^{n}\left(G_{p}\right)$. 
Pour $1 \leq p$, on définit le module des monomes $w^{I^{l}}$ n'intervenant que dans des nombres caractéristiques nuls si ces nombres sont de la forme $w^{I^{p}} \cdot w^{I^{k}}$, le monome $w^{I^{\prime}}$ divisant le monome $w^{I^{p}}$. Soit plus précisement:

$$
\begin{aligned}
\bar{I}^{l}([X])=\left\{\alpha \in H^{l}\left(G_{p}\right) / \text { si } l \leq p, \quad \forall \beta \in H^{k+p-l}\left(G_{p}\right), \quad \beta\right. \text { de la } \\
\text { forme } \left.\beta=w^{I^{p-l}} \cdot w^{I^{k}}, \tau^{k+p}(\alpha \beta)=0\right\} .
\end{aligned}
$$

Et soit $\bar{A}^{*}([X])=H^{*}\left(G_{p}\right) / \bar{I}^{*}([X])$.

La variété $X$ étant fibré sur la sphère $S^{k}$, l'algèbre $A^{*}([X])$ est engendré par les seules classes de Stiefel Whitney $w_{1}, \ldots, w_{p}$. Ainsi l'inclusion $I^{l}([X]) \subseteq \bar{I}^{l}([X])$, pour $l \leq p$, nous donne:

Proposition (2.2). Pour $l \leq p$, on a la surjection naturelle:

$$
A^{l}([X]) \rightarrow \bar{A}^{l}([X]) \rightarrow 0 .
$$

Au travers de l'algèbre $\bar{A}^{l}$, il est possible de relier les algèbres $A^{*}$ de la fibre et de l'espace total du fibré considéré:

Proposition (2.3). Si $p<k$, tout morphisme naturel d'inclusion d'une fibre $F=f^{-1}\left(s_{0}\right) \subset X$, induit pour tout $j<k$ un morphisme surjectif:

$$
A^{j}([F]) \rightarrow \bar{A}^{j}([X]) \rightarrow 0 .
$$

Démonstration. D'une part la suite exacte de Wang du fibré précédent nous assure que le morphisme $h^{j}: H^{j}(X) \rightarrow H^{j}(F)$, induit par l'inclusion $h: f^{-1}\left(s_{0}\right) \subset X$ est une injection pour $j<k$.

D'autre part, le fibré tangent de la variété $X$ se scinde en:

$$
T(X)=T_{S^{k}}(X) \oplus f^{*} T\left(S^{k}\right),
$$

où $T_{S^{k}}(X)$ désigne le fibré tangent "le long des fibres" du fibré $X$, et $f^{*} T\left(S^{k}\right)$ l'image réciproque par l'application $f$ du fibré tangent á la sphère $S^{k}$.

Ainsi, toute application classifiante du fibré $T_{S^{k}}(X)$ dans la Grassmannienne $G_{p}, \tau: X \rightarrow G_{p}$, induit le diagramme commutatif suivant:

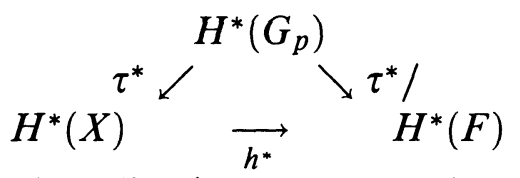

La réstriction $\tau /$ de l'application $\tau$ á la variété $F$ est en effet une application classifiante du fibré tangent á $F$, ceci par construction du fibré $T_{S^{k}}(X)$. 
Désignons par $S W^{*}(X)$ (resp. $S W^{*}(F)$ ) l'image du morphisme $\tau^{*}$ (resp. $\tau^{*} /$ ).

La classe totale de Stiefel Whitney $W\left(T\left(S^{k}\right)\right)$ de la sphère étant égale á $1, S W^{*}(X)$ est bien la sous algèbre de l'algèbre $H^{*}(X)$, engendrée par les classes de Stiefel Whitney de la variété $X$.

Pour $j<k$, le morphisme $h^{j}$ étant injectif et le diagramme (1) étant commutatif, la restriction de $h^{j}$, notée $h^{j} /$, a $S W^{j}(X)$ est un isomorphisme.

Il suffit á présent de vérifier que l'application composée suivante:

$$
S W^{j}(F) \stackrel{\left(h^{\jmath} /\right)^{-1}}{\longrightarrow} S W^{j}(X) \rightarrow \bar{A}^{j}([X]) \rightarrow 0
$$

passe bien au quotient, c'est á dire, vérifier que l'idéal $I^{j}([F])$ s'envoit sur 0 pour $p<k$.

Or pour $j=p<k$, le morphisme $\left(h^{j} /\right)^{-1}$ est un isomorphisme. Pour tout $\alpha \in I^{j}([F]), \alpha$ n'intervient que dans des nombres caractéristiques nuls de la variété $F$. D'où la classe $\left(h^{j} /\right)^{-1}(\alpha)$ n'intervient que dans des diviseurs de degré $p$ nuls des nombres caractéristiques de la variété $X$. Le choix de l'idéal $\bar{I}^{*}([X])$ nous assure du résultat préçédent.

3. Quelques applications dont la fibre théorique a une homologie non finie. Pour toute application $f: X \rightarrow S^{k}, L_{f}$ désigne la fibre théorique de cette application, la variété est supposée de dimension $p+k$. On dira que l'espace $L_{f}$ a une homologie non finie si pour tout entier $N$ il existe un entier $n>N$ tel que l'on ait $H_{n}\left(L_{f}\right) \neq 0$.

Proposition (3.1). S'il existe $j, p+1<j<k-1$, tel que $H_{j}(X)$ soit non nul, alors pour toute application continue $f: X \rightarrow S^{k}$, la fibre théorique de $f$ a une homologie non finie.

Démonstration. La variété $X$ étant de dimension $k+p$, la suite exacte de Wang de la fibration de Serre associée á $f$, dont $L_{f}$ est la fibre nous donne:

$$
\begin{aligned}
& \text { Pour } i>k+p \quad H_{i-k+1}\left(L_{f}\right) \approx H_{i}\left(L_{f}\right) \\
& i<k \quad H_{i-1}\left(L_{f}\right) \approx H_{i-1}(X) .
\end{aligned}
$$

Ainsi si pour $p+1<j<k-1 H_{j}(X) \neq 0$, pour tout entier $m$ on a:

$$
H_{j+m(k-1)}\left(L_{f}\right) \approx H_{j}(X) \neq 0 .
$$


Soit $\omega \in N_{k+p}$, la non nullité du groupe $A^{j}(\omega)$ entraine la non nullité du groupe de cohomologie $H^{j}(X) \approx H_{k+p-j}(X)$ pour toute variété de la classe de cobordisme $\omega$. Ainsi on a:

TheOreme (3.2). Soit $\omega$ un élément du groupe de cobordisme $N_{k+p}$. S'il existe $j, p+1<j<k-1$, avec $A^{j}(\omega) \neq 0$, alors pour toute variété $X$ de la classe $\omega$ et toute application continue $f$ de la variété $X$ dans la sphère $S^{k}$, la fibre théorique de $f$ a une homologie non finie.

Ce théorème et la Proposition (2.1) du paragraphe précédent nous donne:

Proposition (3.3). Soit $\omega \in N_{k+p}, \omega \neq 0$. Si $p<(k-2) / 2$ ou si $p=(k-2) / 2$ pour $p$ impair, pour toute variété $X$ de la classe $\omega$ et toute application continue $f: X \rightarrow S^{k}$, la fibre théorique de $f$ a une homologie non finie.

Proposition (3.4). Soit $\omega \in N_{k+p}, \omega \neq 0$ et l'entier $k+p$ vérifiant l'une des conditions suivantes:

(a) $k+p=2^{s}, s \geq 2, k>2^{s-1}+1$ et $A^{i}(\omega)=0$ pour $0<i \leq 2^{s-2}$

(b) $k+p=5.2^{s}, s \geq 0, k>5.2^{s-1}+1$ et $A^{i}(\omega)=0$ pour $0<i \leq 2^{s}$. Alors pour toute variété $X$ de la classe $\omega$ et toute application continue $f: X \rightarrow S^{k}$, la fibre théorique de $f$ a une homologie non finie.

Démonstration. Dans [6], Théorème 2, R. E. Stong montre que si $k+p=2^{s}$ et $A^{i}(\omega)=0$ pour $0<i \leq 2^{s-2}$, ou si $k+p=5.2^{s}$ et $A^{i}(\omega)=0$ pour $0<i \leq 2^{s}, \omega$ est la classe de $\left(\mathbf{P}_{2}\right)^{2^{s-1}}$ dans le premier cas, celle de $(P(1.2))^{2^{s}}$ dans le second. On désigne par $\mathbf{P}_{2}$ le plan projectif réel et par $P(1.2)$ la variété de Dold de dimension 5.

Le nombre caractéristique $w_{1}^{2}$ est non nul pour le plan projectif, d'où le nombre $w_{2^{s-1}}^{2}$ de la variété $\left(\mathbf{P}_{2}\right)^{2^{s-1}}$ est non nul. Le groupe $A^{2^{s-1}}\left(\left[\mathbf{P}_{2}^{2^{s-1}}\right]\right)$ étant ainsi non nul, le résultat dans le premier cas résulte du théorème précedent. D'autre part le nombre caractéristique $w_{1} w_{2}^{2}$ du générateur de Dold $P(1.2)$ est non nul. Ainsi le nombre $w_{2^{s}}$. $w_{2^{s+1}}^{2}$ est non nul pour la variété $P(1.2)^{2^{s}}$. De la même maniére que préçedemment, le résultat découle dans le deuxième cas de la non nullité du groupe $A^{2^{s+1}}\left(\left[P(1.2)^{2^{s}}\right]\right)$.

4. Quelques classes de cobordisme refusant de se fibrer sur des spheres. Revenant au problème de Conner et Floyd précédent, la 
Proposition (3.3) nous donne le résultat suivant:

Proposition (4.1). Si $\omega \in N_{k+p}, p<(k-2) / 2$ ou $p=(k-2) / 2$ pour $p$ impair, alors la classe $\omega$ se fibre sur la sphère $S^{k}$ si et seulement si elle est nulle.

Utilisant la Proposition (3.4) et les modules $\bar{A}^{i}$ construits au paragraphe 2 , on peut énoncer le résultat partiel suivant pour $p<k$ :

Theoreme (4.2). Soit $f: X \rightarrow S^{k}$ un fibré $C^{\infty}$, connexe, de fibre une variété compacte $F$. Soit $\omega$ la classe de cobordisme de la variété $X$, on suppose que la classe $\omega$ et l'entier $k+p$ vérifient les conditions suivantes:

(a) $k+p$ impair, $p<k, A^{i}(\omega)=\bar{A}^{i}(\omega)$ pour $0<i \leq 2^{s}$ avec: $k+p<\inf \left\{(s+5) 2^{s+1}, N(s)\right\}$ où

$$
N(s)=\left\{\begin{array}{l}
(s+1) 2^{s+2}+1 \text { pour s pair, } \\
(s+2) 2^{s+2} \text { pour } s \text { impair }
\end{array}\right.
$$

(b) $k+p$ pair, $k+p \neq 2^{s}$ et $5 \cdot 2^{s}, p<k, A^{i}(\omega)=\bar{A}^{i}(\omega)$ pour like $0<i \leq 2^{s}$ avec $k+p<2^{s+3}$

(c) $k+p=2^{s}, s \geq 2, k>2^{2-1}+1, A^{i}(\omega)=\bar{A}^{l}(\omega)$ pour $0<i \leq 2^{s-2}$

(d) $k+p=5 \cdot 2^{s}, s \geq 0, k>5 \cdot 2^{s-1}+1, A^{i}(\omega)=\bar{A}^{i}(\omega)$ pour $0<i \leq 2^{s}$.

Alors si la classe de cobordisme de la variété $F$ est nulle, la classe de cobordisme $\omega$ de l'espace total du fibré est aussi nulle.

Démonstration. Pour $p<k$ par la Proposition $(2.3)$, si $[F]=0$, on a $A^{i}([F])=\bar{A}^{i}(\omega)=0$, pour $i<k$. D'où, sous les hypothèses de la proposition précédente, les groupes $A^{i}(\omega)$ seront nuls pour les conditions sur l'indice $i$ données précédemment.

Les cas (a) et (b) sont alors une conséquence immédiate des résultats de R. E. Stong ([6], Théorèmes 1 et 2). Les cas (c) et (d) découlent de la Proposition (3.4).

Remarque (4.3). Pour des indices $i \leq p$, la condition $A^{i}(\omega)=$ $\bar{A}^{i}(\omega)$ est liée á l'existence, pour la classe $\omega$, de nombres caractéristiques non nuls ne provenant pas d'un produit de monomes de degré $p$ et de degré $k$.

En particulier, si tous les nombres caractéristiques non nuls de la classe $\omega$ sont divisibles par un monome de degré $p$, pour tout $i \leq p$ on aura l'égalité $A^{i}(\omega)=\bar{A}^{i}(\omega)$. 
Corollaire (4.4). Soit $\omega$ et $k+p$ vérifiant les hypothèses du théorème précédent, et $X$ une variété connexe de la classe $\omega$. Soit $f: X \rightarrow S^{k}$ une application $C^{\infty}$ telle que l'image réciproque d'une valeur régulière soit une variété bord.

Alors $f$ est cobordante á une fibration $C^{\infty}$ connexe, si et seulement si la classe $\omega$ est nulle.

Remarque (4.5). Dans le Théorème (4.2) et le Corollaire (4.4), l'hypothèse de connexité du fibré est nécessaire pour utiliser les algèbres $A^{*}$. Il n'est donc pas possible de se ramener au cas d'un fibré de fibre une variété bord par un procédé de réunion disjointe. Cependant on a le corollaire suivant:

Corollaire (4.6). Sous les hypothèses du théorème précédent, si de plus le fibré considéré $f: X \rightarrow S^{k}$ possède une section $s: S^{k} \rightarrow X$, telle que le fibré normal á $s(X)$ dans $X$ soit trivial, alors la classe de $f$ dans le groupe de cobordisme $N_{k+p}\left(S^{k}\right)$ est égale á celle de la projection $F \times S^{k} \rightarrow S^{k}$.

Démonstration. Pour pouvoir se ramener aux hypothèses de la proposition précédente, il nous faut un fibré $C^{\infty}$ connexe, de fibre la somme connexe de deux exemplaires de la variété $F$, fibré qui soit cobordant á la réunion disjointe des fibrés $f: X \rightarrow S^{k}$ et $F \times S^{k} \rightarrow S^{k}$. Si $f$ a une section dont le fibré normal est trivial, la construction de R. L. W. Brown ([1], Lemme 5.1) nous donne le fibré connexe cherché.

\section{BIBLIOGRAPHIE}

[1] R. L. W. Brown, Cobordism and bundles over spheres, Michigan Math. J., 16 (1969), 315-320.

[2] P. E. Conner and E. E. Floyd, Fibering within a cobordism class, Michigan Math. J., 12 (1965), 33-47.

[3] A. Didierjean, Classes de cohomologie invariantes par cobordisme et classes de cobordisme se fibrant sur des sphères, C. R. Acad. Sc. Paris, 289 (1979), 165167.

[4] S. M. Kahn, Cobordism obstructions to fibering manifolds over spheres, Pacific J. Math., 114 (1984).

[5] D. F. X. O'Reilly, Cobordism classes of fiber bundles, Pacific J. Math., 69 (1977), 467-475.

[6] R. E. Stong, Cobordism and Stiefel Whitney number, Topology, 4 (1965), $241-$ 256. 
Centro de Investigation y de Estudios Avandos Del I.P.N Apartado Postal 14-740

07000 MeXico 14 D.F.

AND

UNiversite Louis PAsteur

7 RUE RENE DESCARTES

67084 Strasbourg Cedex, France 



\title{
PACIFIC JOURNAL OF MATHEMATICS EDITORS
}

\author{
V. S. VARADARAJAN \\ (Managing Editor) \\ University of California \\ Los Angeles, CA 90024 \\ HERBERT Clemens \\ University of Utah \\ Salt Lake City, UT 84112 \\ R. FINN \\ Stanford University \\ Stanford, CA 94305
}

\author{
HERMANN FLASCHKA \\ University of Arizona \\ Tucson, AZ 85721
}

Ramesh A. Gangolli University of Washington Seattle, WA 98195

VAUGHAN F. R. JONES University of California Berkeley, CA 94720
ROBION KIRBY

University of California

Berkeley, CA 94720

C. C. MOORE

University of California

Berkeley, CA 94720

HAROLD STARK

University of California, San Diego La Jolla, CA 92093

\section{ASSOCIATE EDITORS}

\author{
R. ARENS \\ E. F. BECKENBACH \\ B. H. NEUMANN \\ F. WOLF \\ K. YOSHIDA \\ (1906-1982)

\section{SUPPORTING INSTITUTIONS}

\section{UNIVERSITY OF ARIZONA} \\ UNIVERSITY OF BRITISH COLUMBIA \\ CALIFORNIA INSTITUTE OF TECHNOLOGY \\ UNIVERSITY OF CALIFORNIA \\ MONTANA STATE UNIVERSITY \\ UNIVERSITY OF NEVADA, RENO \\ NEW MEXICO STATE UNIVERSITY \\ OREGON STATE UNIVERSITY \\ UNIVERSITY OF OREGON \\ UNIVERSITY OF SOUTHERN CALIFORNIA \\ STANFORD UNIVERSITY \\ UNIVERSITY OF HAWAII \\ UNIVERSITY OF TOKYO \\ UNIVERSITY OF UTAH \\ WASHINGTON STATE UNIVERSITY \\ UNIVERSITY OF WASHINGTON
}

The Supporting Institutions listed above contribute to the cost of publication of this Journal, but they are not owners or publishers and have no responsibility for its content or policies.

Mathematical papers intended for publication in the Pacific Journal of Mathematics should be in typed form or offset-reproduced (not dittoed), double spaced with large margins. Please do not use built up fractions in the text of the manuscript. However, you may use them in the displayed equations. Underline Greek letters in red, German in green, and script in blue. The first paragraph must be capable of being used separately as a synopsis of the entire paper. In particular it should contain no bibliographic references. Please propose a heading for the odd numbered pages of less than 35 characters. Manuscripts, in triplicate, may be sent to any one of the editors. Please classify according to the scheme of Math. Reviews, Index to Vol. 39. Supply name and address of author to whom proofs should be sent. All other communications should be addressed to the managing editor, or Elaine Barth, University of California, Los Angeles, California 90024

There are page-charges associated with articles appearing in the Pacific Journal of Mathematics. These charges are expected to be paid by the author's University, Government Agency or Company. If the author or authors do not have access to such Institutional support these charges are waived. Single authors will receive 50 free reprints; joint authors will receive a total of 100 free reprints. Additional copies may be obtained at cost in multiples of 50 .

The Pacific Journal of Mathematics is issued monthly as of January 1966. Regular subscription rate: $\$ 190.00$ a year (5 Vols., 10 issues). Special rate: $\$ 95.00$ a year to individual members of supporting institutions.

Subscriptions, orders for numbers issued in the last three calendar years, and changes of address should be sent to Pacific Journal of Mathematics, P.O. Box 969, Carmel Valley, CA 93924, U.S.A. Old back numbers obtainable from Kraus Periodicals Co., Route 100, Millwood, NY 10546.

The Pacific Journal of Mathematics at P.O. Box 969, Carmel Valley, CA 93924 (ISSN 0030-8730) publishes 5 volumes per year. Application to mail at Second-class postage rates is pending at Carmel Valley, California, and additional mailing offices. Postmaster: send address changes to Pacific Journal of Mathematics, P.O. Box 969, Carmel Valley, CA 93924.

PUBLISHED BY PACIFIC JOURNAL OF MATHEMATICS, A NON-PROFIT CORPORATION Copyright (C) 1988 by Pacific Journal of Mathematics 


\section{Pacific Journal of Mathematics}

\section{Vol. 132, No. $2 \quad$ February, 1988}

Jeffery Marc Bergen and Luisa Carini, A note on derivations with power central values on a Lie ideal ..............................209

Alfonso Castro and Sumalee Unsurangsie, A semilinear wave equation

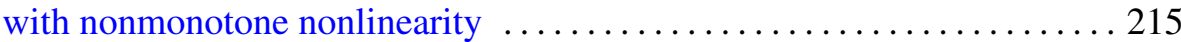

Marius Dadarlat, On homomorphisms of matrix algebras of continuous

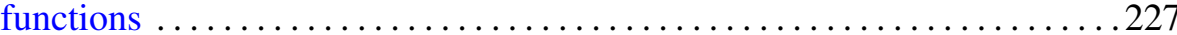

A. Didierjean, Quelques classes de cobordisme non orienté refusant de se



Edward George Effros and Zhong-Jin Ruan, On matricially normed spaces

Sherif El-Helaly and Taqdir Husain, Orthogonal bases are Schauder bases

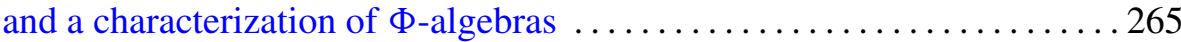

Edward Richard Fadell and Peter N-S Wong, On deforming $G$-maps to be

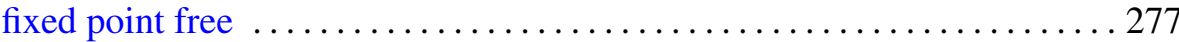

Jean-Jacques Gervais, Stability of unfoldings in the context of equivariant



Douglas Martin Grenier, Fundamental domains for the general linear group

Ronald Scott Irving and Brad Shelton, Loewy series and simple projective

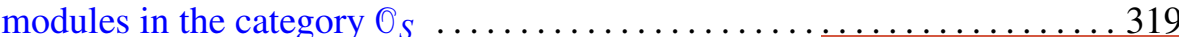

Russell Allan Johnson, On the Sato-Segal-Wilson solutions of the K-dV equation

Thomas Alan Keagy and William F. Ford, Acceleration by subsequence transformations

Min Ho Lee, Mixed cusp forms and holomorphic forms on elliptic varieties

Charles Livingston, Indecomposable surfaces in 4-space 